\title{
Semiblind Channel Estimation and Equalization for MIMO Space-Time Coded OFDM
}

\author{
Yonghong Zeng, Senior Member, IEEE, W. H. Lam, and Tung Sang Ng, Fellow, IEEE
}

\begin{abstract}
Based on the special features of space-time coding (STC) and orthogonal frequency-division multiplexing (OFDM), it is mathematically proved that all channel responses of a multiple-input-multiple-output STC-OFDM system can be identified blindly subject to two ambiguity matrices with a subspace-based method. A method is then presented to resolve the two ambiguity matrices by using few pilot symbols. With the estimated channels, a frequency domain approach is presented to recover the transmitted symbols. The presented semiblind algorithms are valid even if the channel transfer functions are not coprime and do not require precise channel order information (only an upper bound for the orders is required).
\end{abstract}

Index Terms-Blind, channel estimation, equalization, multipleinput-multiple-output (MIMO), multiuser, orthogonal frequencydivision multiplexing (OFDM), semiblind, space-time coding (STC), subspace.

\section{INTRODUCTION}

B Y USING multiple transmit antennas and single or multiple receive antennas, space-time coding (STC) has the ability to greatly reduce the bit error rate (BER) or increase the data rate. Therefore, STC has been recognized as a promising technique for wideband wireless communication [1], [2]. So far, a number of STC schemes have been proposed [3]-[9]. Among them, some schemes assume a flat-fading channel condition to achieve their claimed performances [3], [8], [9], and the others can work well for frequency-selective multipath channels [4]-[7]. Orthogonal frequency-division multiplexing (OFDM) has also emerged as a major technique in the future fourth generation (4G) communications [10]. Properly combining the STC and OFDM produces the STC-OFDM which not only keeps the diversity gain of STC but also enjoys the advantages of OFDM such as multipath mitigation and fast frequency domain equalization. An example of such a combination is the STC-OFDM proposed in [7], which uses two transmit antennas and one receive antenna, and achieves a diversity gain of order two for frequency-selective channels [11]. Although

Manuscript received July 7, 2004; revised March 28, 2005 and June 27, 2005. This work was supported in part by Grant HKU 7168/03E from the Research Grants Council of the Hong Kong SAR, China, and in part by A*STAR EHS Research Grant 0221060041, Singapore. This paper was presented in part at the IEEE International Conference on Communications (ICC 2004), Paris, France. This paper was recommended by Associate Editor W.-S. Lu.

Y. H. Zeng was with the Department of Electrical and Electronic Engineering, The University of Hong Kong, Hong Kong. He is now with the Institute for Infocomm Research, A-STAR, Singapore (e-mail: yhzeng@ieee.org).

W. H. Lam and T. S. Ng are with the Department of Electrical and Electronic Engineering, The University of Hong Kong, Hong Kong (e-mail: whlam@eee.hku.hk; tsng@eee.hku.hk).

Digital Object Identifier 10.1109/TCSI.2005.856671 some enhancements to the STC, such as the unitary or differential STC [12]-[15], can dispense with channel estimation for flat-fading channels, they either have considerable performance losses relative to coherent detection or require a prohibitive high computational complexity. Hence, in general, channel state information (CSI) is required for a STC system to achieve its full capacity, especially when the channels are frequency selective with multipath.

Channel estimation is usually achieved by a training method or a blind method or their combination. A training method is simple but it consumes some bandwidth which is very precious in wireless communications [16]. A blind method is usually more complex but it costs zero or very little bandwidth. Although there have been extensive research works on blind methods, most of them do not consider the STC structure (see [17] and [18], and the references herein). Among the blind methods for the STC systems, most of them can only deal with flat-fading channels [19], [20]. There have been only few works on the STC-OFDM [7], [11], [21], [22]. In [7], a deterministic blind method is presented, which requires the channel transfer functions to be coprime (no common zeros) and the transmitted signals to have constant-modulus (CM). In [11], a subspace-based blind method is proposed for a precoded STC-OFDM. It should be noted that the additional precoding step not only increase the system complexity but also consumes additional bandwidth. In [21] and [23], blind or semiblind equalization methods for general space-time coded systems are considered. Unfortunately, the identifiability of the methods are in question. The blind method in [21] is extended by [22] to the MIMO-OFDM when the cyclic prefix (CP) length is shorter than the channel length. The methods in [7], [11] are only valid for single user case with two transmitting antennas and one receiving antenna. When multiple users (virtual users) share the same frequency band and each user uses the STC, a multiple-input-multiple-output (MIMO) STC system is created. Channel estimation in an MIMO STC system is usually much more difficult because the number of channels increases rapidly with the number of antennas and users. Because of the STC, the channel estimation and equalization methods for the MIMO-OFDM in [24] cannot be used here. Especially the channel identifiability for the MIMO STC-OFDM is quite different from that of the MIMO-OFDM.

In this paper, an MIMO STC-OFDM system extended from the system in [7] is considered. We assume that the zero-padding OFDM (ZP-OFDM) [25] other than the classical cyclic-prefixed OFDM (CP-OFDM) is used in the system, because the ZP-OFDM not only has all the advantages of CP-OFDM but also simplifies channel estimation [18], [24]-[26]. It is proved 


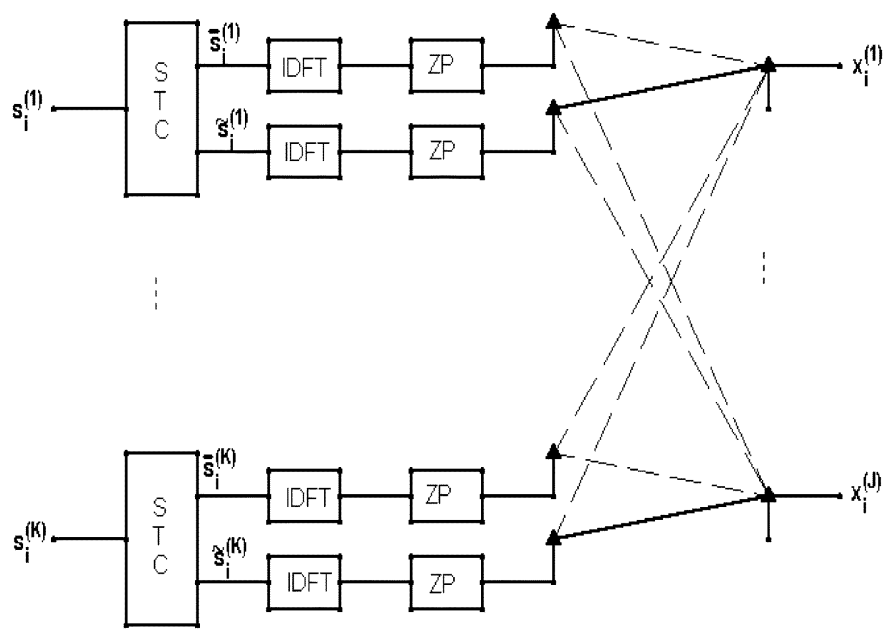

Fig. 1. Multiuser MIMO STC-OFDM system.

mathematically that all channel responses of the MIMO STCOFDM system can be identified blindly subject to two ambiguity matrices with a subspace-based method, if the transmitted symbol is real (for example, PAM constellation) or symmetric. The method does not need precoding or CM modulating and works well even if the channel transfer functions are not coprime. The two ambiguity matrices remaining undetermined are inherent to any second-order statistics (SOS) based method. To determine the two matrices, a method which uses few pilot symbols is proposed. With the estimated channels, a frequency domain approach is then presented to recover the transmitted symbols. Simulations show that the methods are effective and robust. The major drawback of the method is: it requires the input symbols to be real or symmetric.

The rest of the paper is organized as follows. In Section II, the MIMO STC-OFDM system model is described. Section III proves the channel identifiability and gives a practical subspacebased algorithm. Equalization is discussed in Section IV. The method to resolve the ambiguities through the use of pilot symbols is considered in Section V. Some simulation results and discussions are given in Section VI. Finally, conclusions are drawn in Section VI.

Some notations are used in the following. Superscripts $T, \dagger$ and $*$ stand for transpose, transconjugate (Hermitian), and conjugate, respectively. $\mathbf{I}_{q}$ is the identity matrix of order $q$.

\section{MIMO STC-OFDM MODEL}

A STC-OFDM system with two transmitting antenna and one receiving antenna was proposed in [7]. The system can achieve diversity gain of order two if exact channel responses are known [11]. If there are $K$ users (or virtual users, that is, different data streams of the same user), each user uses the STC-OFDM and $J(J \geq K)$ antennas are installed for receiving, an MIMO STC-OFDM system (with $2 K$ transmitting antennas and $J$ receiving antennas) is created as shown in Fig. 1. Such a system can be used for both uplink and downlink. A practical example for downlink is: the base-station divides the data stream of an user into $K$ substreams (virtual users) and these substreams are transmitted simultaneously on the same frequency band via the
STC-OFDM. In the mobile end, at least $K$ antennas must be installed such that recovering the transmitted signal is possible. Such a system can increase the data rate by $K$ times and, therefore, is useful for wideband communications.

Let $\mathbf{s}_{i}^{(k)}=\left[\begin{array}{lll}s_{i}^{(k)}(0) & \cdots & s_{i}^{(k)}(N-1)\end{array}\right]^{T}$ be the block symbol of user $k$ at time $i$ to be transmitted and its inverse discrete Fourier transform (IDFT) is denoted as $\mathbf{u}_{i}^{(k)}=$ $\left[\begin{array}{llll}u_{i}^{(k)}(0) & u_{i}^{(k)}(1) & \cdots & u_{i}^{(k)}(N-1)\end{array}\right]^{T}$. The block symbols are first encoded by the STC [7]. The STC turns the block $\mathbf{s}_{i}^{(k)}$ into two blocks $\overline{\mathbf{s}}_{i}^{(k)}$ and $\tilde{\mathbf{s}}_{i}^{(k)}$, where

$$
\begin{aligned}
& \overline{\mathbf{s}}_{2 i}^{(k)}=\mathbf{s}_{2 i}^{(k)}, \quad \overline{\mathbf{s}}_{2 i+1}^{(k)}=-\left(\mathbf{s}_{2 i+1}^{(k)}\right)^{*} \\
& \tilde{\mathbf{s}}_{2 i}^{(k)}=\mathbf{s}_{2 i+1}^{(k)}, \quad \tilde{\mathbf{s}}_{2 i+1}^{(k)}=\left(\mathbf{s}_{2 i}^{(k)}\right)^{*} .
\end{aligned}
$$

Two transmitters $\left(\mathrm{TX}_{2 k-1}\right.$ and $\left.\mathrm{TX}_{2 k}\right)$ are used to transmit the blocks $\overline{\mathbf{s}}_{i}^{(k)}$ and $\tilde{\mathbf{s}}_{i}^{(k)}$, respectively. Before transmitting, the blocks are further modulated by the OFDM. We assume that the ZP-OFDM [18], [25] instead of the CP-OFDM is used, because the ZP-OFDM avoids interblock interference (IBI) and, therefore, simplifies channel estimation and equalization [18], [24]-[26]. In ZP-OFDM, a block symbol is transformed by the IDFT, and then $L(L \leqslant N)$ zeros are added to the tail of the transformed block (zero-padding), where cyclic prefix is no longer needed. Let $h^{(j, k)}(l)\left(l=0,1, \ldots, L_{j, k}\right)$ be the channel response (including the transmitting and receiving filters) from transmitter $\mathrm{TX}_{k}$ to receiver $\mathrm{RX}_{j}$, where $L_{j, k}$ is the channel order and $L_{j, k} \leqslant L(k=1,2, \ldots, 2 K, j=1,2, \ldots, J)$. Let $\mathbf{x}_{i}^{(j)}=\left[\begin{array}{lll}x_{i}^{(j)}(0) & \cdots & x_{i}^{(j)}(M-1)\end{array}\right]^{T}(M=N+L)$ be the length- $M$ block symbol received at antenna $j$ and $\mathbf{x}_{i}(n)=\left[\begin{array}{lll}x_{i}^{(1)}(n) & \cdots & x_{i}^{(J)}(n)\end{array}\right]^{T}$. In the following, we consider two types of inputs: real and symmetric.

1) If the inputs are real, we have

$$
\begin{aligned}
\mathbf{x}_{2 i}(n)= & \sum_{l=0}^{L} \mathbf{h}_{1}(l) \mathbf{u}_{2 i}(n-l)+\sum_{l=0}^{L} \mathbf{h}_{2}(l) \mathbf{u}_{2 i+1}(n-l) \\
& +\boldsymbol{\eta}_{2 i}(n), \quad n=0,1, \ldots, M-1 \\
\mathbf{x}_{2 i+1}(n)= & -\sum_{l=0}^{L} \mathbf{h}_{1}(l) \mathbf{u}_{2 i+1}(n-l)+\sum_{l=0}^{L} \mathbf{h}_{2}(l) \mathbf{u}_{2 i}(n-l) \\
& +\boldsymbol{\eta}_{2 i+1}(n), \quad n=0,1, \ldots, M-1
\end{aligned}
$$

where

$$
\begin{aligned}
\mathbf{u}_{i}(n)= & {\left[\begin{array}{llll}
u_{i}^{(1)}(n) & \ldots & u_{i}^{(K)}(n)
\end{array}\right]^{T} } \\
\mathbf{h}_{1}(l)= & {\left[\begin{array}{cccc}
h^{(1,1)}(l) & h^{(1,3)}(l) & \ldots & h^{(1,2 K-1)}(l) \\
\vdots & & & \vdots \\
h^{(J, 1)}(l) & h^{(J, 3)}(l) & \ldots & h^{(J, 2 K-1)}(l)
\end{array}\right] } \\
\mathbf{h}_{2}(l)= & {\left[\begin{array}{cccc}
h^{(1,2)}(l) & h^{(1,4)}(l) & \ldots & h^{(1,2 K)}(l) \\
\vdots & & & \vdots \\
h^{(J, 2)}(l) & h^{(J, 4)}(l) & \ldots & h^{(J, 2 K)}(l)
\end{array}\right] }
\end{aligned}
$$


and $\boldsymbol{\eta}_{i}(n)$ is the channel noise. Note that $\mathbf{u}_{i}(n)=0$, if $n<0$ or $N \leqslant n<M$. Now let

$$
\begin{aligned}
& \mathbf{x}_{i}=\left[\begin{array}{c}
\mathbf{x}_{i}(0) \\
\mathbf{x}_{i}(1) \\
\vdots \\
\mathbf{x}_{i}(M-1)
\end{array}\right] \\
& \mathbf{u}_{i}=\left[\begin{array}{c}
\mathbf{u}_{i}(0) \\
\mathbf{u}_{i}(1) \\
\vdots \\
\mathbf{u}_{i}(N-1)
\end{array}\right] \\
& \boldsymbol{\eta}_{i}=\left[\begin{array}{c}
\boldsymbol{\eta}_{i}(0) \\
\boldsymbol{\eta}_{i}(1) \\
\vdots \\
\boldsymbol{\eta}_{i}(M-1)
\end{array}\right] \\
& \mathbf{H}_{j}=\left[\begin{array}{ccccc}
\mathbf{h}_{j}(0) & & & & \\
\vdots & \mathbf{h}_{j}(0) & & & \\
\mathbf{h}_{j}(L) & \vdots & \ddots & & \\
& \mathbf{h}_{j}(L) & & \ddots & \\
& & \ddots & & \mathbf{h}_{j}(0) \\
& & & \ddots & \vdots \\
& & & & \mathbf{h}_{j}(L)
\end{array}\right], \quad j=1,2
\end{aligned}
$$

where $\mathbf{H}_{1}$ and $\mathbf{H}_{2}$ are $J M \times K N$ lower triangular block Toeplitz matrix. Then (1) and (2) can be written as

$$
\begin{aligned}
\mathbf{x}_{2 i} & =\mathbf{H}_{1} \mathbf{u}_{2 i}+\mathbf{H}_{2} \mathbf{u}_{2 i+1}+\boldsymbol{\eta}_{2 i}, \quad i=0,1, \ldots \\
\mathbf{x}_{2 i+1} & =-\mathbf{H}_{1} \mathbf{u}_{2 i+1}+\mathbf{H}_{2} \mathbf{u}_{2 i}+\boldsymbol{\eta}_{2 i+1}, \quad i=0,1, \ldots
\end{aligned}
$$

Let

$$
\begin{aligned}
\mathbf{r}_{i} & =\left[\begin{array}{c}
\mathbf{x}_{2 i} \\
\mathbf{x}_{2 i+1}
\end{array}\right] \\
\mathbf{v}_{i} & =\left[\begin{array}{c}
\mathbf{u}_{2 i} \\
\mathbf{u}_{2 i+1}
\end{array}\right] \\
\boldsymbol{\xi}_{i} & =\left[\begin{array}{c}
\boldsymbol{\eta}_{2 i} \\
\boldsymbol{\eta}_{2 i+1}
\end{array}\right] \\
\mathbf{H} & =\left[\begin{array}{cc}
\mathbf{H}_{1} & \mathbf{H}_{2} \\
\mathbf{H}_{2} & -\mathbf{H}_{1}
\end{array}\right] .
\end{aligned}
$$

Then (6) and (7) become

$$
\mathbf{r}_{i}=\mathbf{H v}_{i}+\boldsymbol{\xi}_{i}, \quad i=0,1, \ldots
$$

2 If the inputs are symmetric, that is, $s_{i}^{(k)}(n)=$ $s_{i}^{(k)}(N-n), n=1,2, \ldots, N / 2-1$, (1) and (2) should be revised as (noting that the IDFT of $s_{i}^{(k)}$ is also symmetric)

$$
\begin{aligned}
\mathbf{x}_{2 i}(n)= & \sum_{l=0}^{L} \mathbf{h}_{1}(l) \mathbf{u}_{2 i}(n-l)+\sum_{l=0}^{L} \mathbf{h}_{2}(l) \mathbf{u}_{2 i+1}(n-l) \\
& +\boldsymbol{\eta}_{2 i}(n) \\
\mathbf{x}_{2 i+1}^{*}(n)= & -\sum_{l=0}^{L} \mathbf{h}_{1}^{*}(l) \mathbf{u}_{2 i+1}(n-l)+\sum_{l=0}^{L} \mathbf{h}_{2}^{*}(l) \mathbf{u}_{2 i}(n-l) \\
& +\boldsymbol{\eta}_{2 i+1}^{*}(n) .
\end{aligned}
$$

Amending the notations as

$$
\mathbf{r}_{i}=\left[\begin{array}{c}
\mathbf{x}_{2 i} \\
\mathbf{x}_{2 i+1}^{*}
\end{array}\right], \quad \boldsymbol{\xi}_{i}=\left[\begin{array}{c}
\boldsymbol{\eta}_{2 i} \\
\boldsymbol{\eta}_{2 i+1}^{*}
\end{array}\right], \quad \mathbf{H}=\left[\begin{array}{cc}
\mathbf{H}_{1} & \mathbf{H}_{2} \\
\mathbf{H}_{2}^{*} & -\mathbf{H}_{1}^{*}
\end{array}\right]
$$

we also obtain the (9).

Another technique [4], the combination of STC and the single-carrier frequency-domain equalization, has nearly the same advantage of the STC-OFDM, but avoids OFDMs shortcomings of high peak-to-average power ratio and high sensitivity to frequency errors. If the system is extended to multiuser and $\mathrm{CP}$ for each symbol block is replaced by $\mathrm{ZP}$ as for the ZP-OFDM, the received signals can be expressed as

$$
\mathbf{r}_{i}=\mathbf{H}\left[\begin{array}{c}
\mathbf{s}_{2 i} \\
\mathbf{s}_{2 i+1}
\end{array}\right]+\xi_{i}, \quad i=0,1, \ldots
$$

Hence, a channel estimation method for an MIMO STC-OFDM is also valid for such a system.

\section{BLIND ChanNel Estimation}

In this section, blind estimation of the channels using the subspace technique is considered. Subspace technique is wellknown in signal processing and has been widely used in communication [11], [17], [18], [24], [27]-[30]. The principle of the subspace technique is that the signal subspace (or noise subspace) can be determined from the SOS of the output samples. The signal subspace is the range space of matrix $\mathbf{H}$ (denoted by $\operatorname{span}(\mathbf{H})$, which is all the possible linear combinations of the column vectors of $\mathbf{H}$ ), if the problem can be modeled as (9). Many different problems can be turned into a similar model as (9), but the matrix $\mathbf{H}$ varies from problem to problem. The difficulty in using the subspace technique is on two aspects, that is, to prove if the channels can be identified from the known signal subspace or not, and how to obtain the channels if they are identifiable. Based on the special structure of the MIMO STC-OFDM, which gives the special matrix $\mathbf{H}$, we will prove that here the channels are identifiable from the signal subspace up to two ambiguity matrices and give a practical method to find the channels.

The following statistical properties of the transmitted symbols $s_{l}^{(k)}(n)$ and channel noise $\eta_{i}^{(j)}(n)$ are assumed.

(A1) Noises are white and uncorrelated, that is

$$
\mathrm{E}\left(\boldsymbol{\eta}_{i}^{(j)}(n)\left(\eta_{l}^{(k)}(m)\right)^{*}\right)= \begin{cases}\sigma_{\eta}^{2} & (i, j, n)=(l, k, m) \\ 0, & (i, j, n) \neq(l, k, m) .\end{cases}
$$


(A2) Noises and transmitted signals are uncorrelated, that is, $\mathrm{E}\left(\boldsymbol{\eta}_{i}^{(j)}(n)\left(s_{l}^{(k)}(m)\right)^{*}\right)=0$.

Here $\mathrm{E}(y)$ means the mathematical expectation of a random variable $y$. It is also assumed that $J \geq K$.

\section{A. Identifiability}

Based on the assumptions (A1 and A2), the statistical autocorrelation matrices of $\mathbf{r}_{i}$ can be written as

$$
\mathbf{R}_{r}=\mathrm{E}\left(\mathbf{r}_{i} \mathbf{r}_{i}^{\dagger}\right)=\mathbf{H R}_{v} \mathbf{H}^{\dagger}+\sigma_{\eta}^{2} \mathbf{I}_{2 M}
$$

where $\mathbf{R}_{v}=\mathrm{E}\left(\mathbf{v}_{i} \mathbf{v}_{i}^{\dagger}\right)$ is a positive definite matrix. The smallest eigenvalue of matrix $\mathbf{R}_{r}$ is $\sigma_{\eta}^{2}$. If $\mathbf{H}$ is of full column rank, the rank of $\mathbf{H R}_{v} \mathbf{H}^{\dagger}$ is $2 K N$. Thus, there are $q=2(J M-K N)$ co-orthogonal eigenvectors corresponding to the smallest eigenvalue. These eigenvectors are denoted by $\boldsymbol{\beta}_{k}(k=0,1, \ldots, q-1)$. Based on a simple mathematical derivation which is used in the standard subspace method [17], [18], [27], [28], it is known that

$$
\boldsymbol{\beta}_{k}^{\dagger} \mathbf{H}=0, \quad k=0,1, \ldots, q-1
$$

that is, $\boldsymbol{\beta}_{k}(k=0,1, \ldots, q-1)$ span the left null space of $\mathbf{H}$. Having known the left null space, we can determine the range space $\operatorname{span}(\mathbf{H})$. Equivalently, we can also treat (15) as $2 q K N$ linear equations with $h^{(j, k)}(l)$ as unknowns. For $N \geq L$, the number of equations is usually larger than that of the unknowns. However, since many of the linear equations may be dependent, it is hard to say if the unknowns are identifiable or not. The identifiability of the unknowns is very dependent on the structure of the matrix $\mathbf{H}$.

Lemma 1: There exist constant permutation matrices $\mathbf{P}$ and Q such that

$$
\mathbf{P H Q}=\mathbf{F}
$$

with

$$
\mathbf{F}=\left[\begin{array}{ccccc}
\mathbf{F}(0) & & & & \\
\vdots & \mathbf{F}(0) & & & \\
\mathbf{F}(L) & \vdots & \ddots & & \\
& \mathbf{F}(L) & & \ddots & \\
& & \ddots & & \mathbf{F}(0) \\
& & & \ddots & \vdots \\
& & & & \mathbf{F}(L)
\end{array}\right]
$$

where, for real inputs

$$
\mathbf{F}(l)=\left[\begin{array}{cc}
\mathbf{h}_{1}(l) & \mathbf{h}_{2}(l) \\
\mathbf{h}_{2}(l) & -\mathbf{h}_{1}(l)
\end{array}\right]
$$

and, for symmetric inputs

$$
\mathbf{F}(l)=\left[\begin{array}{cc}
\mathbf{h}_{1}(l) & \mathbf{h}_{2}(l) \\
\mathbf{h}_{2}^{*}(l) & -\mathbf{h}_{1}^{*}(l)
\end{array}\right] .
$$

Lemma 2: If $\mathbf{F}(0)$ is of full column rank, $\mathbf{F}$ is of full column rank.

It is easy to prove the two lemmas. A more general result of Lemma 2 is also proved in [24].
Theorem 1: Let $\mathbf{h}_{j}(l)$ and $\hat{\mathbf{h}}_{j}(l)$ be two MIMO channels, and $\mathbf{H}_{j}$ and $\hat{\mathbf{H}}_{j}$ be defined as (5) respectively from $\mathbf{h}_{j}(l)$ and $\hat{\mathbf{h}}_{j}(l)(j=1,2)$. For real inputs

$$
\mathbf{H}=\left[\begin{array}{cc}
\mathbf{H}_{1} & \mathbf{H}_{2} \\
\mathbf{H}_{2} & -\mathbf{H}_{1}
\end{array}\right], \quad \hat{\mathbf{H}}=\left[\begin{array}{cc}
\hat{\mathbf{H}}_{1} & \hat{\mathbf{H}}_{2} \\
\hat{\mathbf{H}}_{2} & -\hat{\mathbf{H}}_{1}
\end{array}\right]
$$

and for symmetric inputs

$$
\mathbf{H}=\left[\begin{array}{cc}
\mathbf{H}_{1} & \mathbf{H}_{2} \\
\mathbf{H}_{2}^{*} & -\mathbf{H}_{1}^{*}
\end{array}\right], \quad \hat{\mathbf{H}}=\left[\begin{array}{cc}
\hat{\mathbf{H}}_{1} & \hat{\mathbf{H}}_{2} \\
\hat{\mathbf{H}}_{2}^{*} & -\hat{\mathbf{H}}_{1}^{*}
\end{array}\right] .
$$

Let $\hat{\mathbf{F}}(l)$ and $\hat{\mathbf{F}}$ be defined similar to $\mathbf{F}(l)$ and $\mathbf{F}$ in (18) (for real inputs) or in (19) (for symmetric inputs) with $\mathbf{h}_{j}(l)$ replaced by $\hat{\mathbf{h}}_{j}(l)$. Assume that $\mathbf{F}(0)$ and $\hat{\mathbf{F}}(0)$ are of full rank. If $\operatorname{span}(\mathbf{H})=\operatorname{span}(\hat{\mathbf{H}})$, there exist two $K \times K$ constant matrices $\mathbf{b}_{1}$ and $\mathbf{b}_{2}$ such that, for real inputs

$$
\begin{aligned}
& \hat{\mathbf{h}}_{1}(l)=\mathbf{h}_{1}(l) \mathbf{b}_{1}-\mathbf{h}_{2}(l) \mathbf{b}_{2} \\
& \hat{\mathbf{h}}_{2}(l)=\mathbf{h}_{1}(l) \mathbf{b}_{2}+\mathbf{h}_{2}(l) \mathbf{b}_{1}, \quad l=0,1, \ldots, L
\end{aligned}
$$

and, for symmetric inputs

$$
\begin{aligned}
& \hat{\mathbf{h}}_{1}(l)=\mathbf{h}_{1}(l) \mathbf{b}_{1}-\mathbf{h}_{2}(l) \mathbf{b}_{2}^{*} \\
& \hat{\mathbf{h}}_{2}(l)=\mathbf{h}_{1}(l) \mathbf{b}_{2}+\mathbf{h}_{2}(l) \mathbf{b}_{1}^{*}, \quad l=0,1, \ldots, L .
\end{aligned}
$$

Proof: It is easy to verify that $\operatorname{span}(\mathbf{H})=\operatorname{span}(\hat{\mathbf{H}})$ if and only if there exists a $2 K N \times 2 K N$ invertible matrix $\mathbf{B}$ such that

$$
\hat{\mathbf{H}}=\mathbf{H B} \text {. }
$$

Since $\mathbf{P H Q}=\hat{\mathbf{F}}$, we obtain

$$
\mathbf{P}^{T} \hat{\mathbf{F}} Q^{T}=\mathbf{P}^{T} \mathbf{F} \mathbf{Q}^{T} \mathbf{B} .
$$

Therefore,

$$
\hat{\mathbf{F}}=\mathbf{F Q}^{T} \mathbf{B Q} .
$$

Since $\mathbf{Q}^{T} \mathbf{B Q}$ is an invertible matrix, it follows that $\operatorname{span}(\mathbf{F})=$ $\operatorname{span}(\hat{\mathbf{F}})$. Based on the assumptions of the theorem and Lemma $2, \mathbf{F}$ and $\hat{\mathbf{F}}$ are of full column rank. Therefore, from Theorem 1 in [24], there exists a $2 K \times 2 K$ constant invertible matrix b such that

$$
\hat{\mathbf{F}}(l)=\mathbf{F}(l) \mathbf{b}, \quad l=0,1, \ldots, L .
$$

Let

$$
\mathbf{b}=\left[\begin{array}{ll}
\mathbf{b}_{1} & \mathbf{b}_{2} \\
\mathbf{b}_{3} & \mathbf{b}_{4}
\end{array}\right]
$$

where $\mathbf{b}_{j}(j=1,2,3,4)$ are $K \times K$ matrices. In the following, we only consider the case of real inputs. The derivation for the case of symmetric inputs is very similar. Thus,

$$
\begin{array}{r}
{\left[\begin{array}{cc}
\hat{\mathbf{h}}_{1}(l) & \hat{\mathbf{h}}_{2}(l) \\
\hat{\mathbf{h}}_{2}(l) & -\hat{\mathbf{h}}_{1}(l)
\end{array}\right]=\left[\begin{array}{cc}
\mathbf{h}_{1}(l) & \mathbf{h}_{2}(l) \\
\mathbf{h}_{2}(l) & -\mathbf{h}_{1}(l)
\end{array}\right]} \\
\times\left[\begin{array}{ll}
\mathbf{b}_{1} & \mathbf{b}_{2} \\
\mathbf{b}_{3} & \mathbf{b}_{4}
\end{array}\right] .
\end{array}
$$

Comparing the entries of the matrices, we obtain

$$
\begin{aligned}
& \mathbf{h}_{1}(l) \mathbf{b}_{1}+\mathbf{h}_{2}(l) \mathbf{b}_{3}=\mathbf{h}_{1}(l) \mathbf{b}_{4}-\mathbf{h}_{2}(l) \mathbf{b}_{2} \\
& \mathbf{h}_{1}(l) \mathbf{b}_{2}+\mathbf{h}_{2}(l) \mathbf{b}_{4}=\mathbf{h}_{2}(l) \mathbf{b}_{1}-\mathbf{h}_{1}(l) \mathbf{b}_{3} .
\end{aligned}
$$


This equation can be turned to

$$
\mathbf{F}(l)\left[\begin{array}{l}
\mathbf{b}_{1}-\mathbf{b}_{4} \\
\mathbf{b}_{2}+\mathbf{b}_{3}
\end{array}\right]=0
$$

Since $\mathbf{F}(0)$ is assumed to be of full column rank, we have $\mathbf{b}_{1}=$ $\mathbf{b}_{4}$ and $\mathbf{b}_{2}=-\mathbf{b}_{3}$. Thus, (22) is easily obtained from (26) and proof of the theorem is complete.

From this theorem, it is apparent that, using the subspace technique, the channels are identifiable with two ambiguity matrices when only an upper bound is given for the channel orders. The full column rank property of $\mathbf{F}(0)$ is almost surely guaranteed because signal propagation from each of the $2 K$ antennas scattered in the cell is most likely independent. The requirement of coprime channel and CM constraints in [7] are not needed here.

\section{B. Practical Algorithm}

For simplicity, in this subsection and hereafter, we only consider real inputs. Similar results can be obtained for symmetric inputs.

Equation (15) can be expressed equivalently as

$$
\mathbf{H}^{\dagger} \boldsymbol{\beta}_{k}=0, \quad k=0,1, \ldots, q-1 .
$$

Since $\mathbf{H}=\mathbf{P}^{T} \mathbf{F} \mathbf{Q}^{T}$, we have

$$
\mathbf{F}^{\dagger} \mathbf{P} \boldsymbol{\beta}_{k}=0, \quad k=0,1, \ldots, q-1 .
$$

For simplicity, let $\boldsymbol{\alpha}_{k}=\mathbf{P} \boldsymbol{\beta}_{k}$. By dividing the vector $\boldsymbol{\alpha}_{k}$ into blocks as

$$
\boldsymbol{\alpha}_{k}=\left[\begin{array}{llll}
\boldsymbol{\alpha}_{k}^{T}(M-1) & \boldsymbol{\alpha}_{k}^{T}(M-2) & \cdots & \boldsymbol{\alpha}_{k}^{T}(0)
\end{array}\right]^{T}
$$

where $\boldsymbol{\alpha}_{k}(m)(m=0,1, \ldots, M-1)$ are $2 J \times 1$ vectors, it is easy to turn (30) into

$$
\sum_{l=0}^{L} \mathbf{F}^{\dagger}(l) \boldsymbol{\alpha}_{k}(n+L-l)=0, \quad n=0,1, \ldots, N-1
$$

or, equivalently

$$
\sum_{l=0}^{L} \alpha_{k}^{\dagger}(n+L-l) \mathbf{F}(l)=0, \quad n=0,1, \ldots, N-1 .
$$

Denote two matrices $\mathbf{G}_{k}$ and $\overline{\mathbf{F}}$ as

$$
\begin{aligned}
\mathbf{G}_{k} & =\left[\begin{array}{ccccc}
\boldsymbol{\alpha}_{k}^{\dagger}(L) & \boldsymbol{\alpha}_{k}^{\dagger}(L-1) & \cdots & \cdots & \boldsymbol{\alpha}_{k}^{\dagger}(0) \\
\boldsymbol{\alpha}_{k}^{\dagger}(L+1) & \boldsymbol{\alpha}_{k}^{\dagger}(L) & \cdots & \cdots & \boldsymbol{\alpha}_{k}^{\dagger}(1) \\
\vdots & \vdots & & & \vdots \\
\boldsymbol{\alpha}_{k}^{\dagger}(M-1) & \boldsymbol{\alpha}_{k}^{\dagger}(M-2) & \cdots & \cdots & \boldsymbol{\alpha}_{k}^{\dagger}(N-1)
\end{array}\right] \\
\overline{\mathbf{F}} & =\left[\begin{array}{c}
\mathbf{F}(0) \\
\mathbf{F}(1) \\
\vdots \\
\mathbf{F}(L)
\end{array}\right] .
\end{aligned}
$$

Then (33) is equivalent to

$$
\mathbf{G}_{k} \overline{\mathbf{F}}=0, \quad k=0,1, \ldots, q-1 .
$$

Equation (35) can also be expressed as

$$
\mathbf{G} \overline{\mathbf{F}}=0
$$

where $\mathbf{G}$ is a $q N \times 2 J(L+1)$ matrix defined as

$$
\mathbf{G}=\left[\begin{array}{llll}
\mathbf{G}_{0}^{T} & \mathbf{G}_{1}^{T} & \cdots & \mathbf{G}_{q-1}^{T}
\end{array}\right]^{T} .
$$

The unknown channels must satisfy (36). Based on the identifiability proved in the last subsection, the solution of (36) is unique up to two $K \times K$ constant ambiguities matrices $\mathbf{b}_{1}$ and $\mathbf{b}_{2}$. Hence, the dimension size of the solution space (the right null space of $\mathbf{G}$ ) must be $2 K$. Let $\mathbf{V}$ be the matrix whose columns form a basis of the solution space. Then the structured channel matrix $\overline{\mathbf{F}}_{1}$ (the first $K$ columns of matrix $\overline{\mathbf{F}}$ ) should be $\overline{\mathbf{F}}_{1}=\mathbf{V C}$, where $C$ is a $2 K \times K$ ambiguity matrix to be determined. In practice, we can choose the $2 K$ right singular vectors corresponding to the $2 K$ smallest singular values of $\mathbf{G}$ and use them as columns to create the matrix $\mathbf{V}$.

The subspace method (for real inputs) is summarized as follows.

Algorithm 1: Subspace-based Channel

Estimation for STC-OFDM

Step 1.Compute $\mathbf{R}_{r}=\mathrm{E}\left(\mathbf{r}_{i} \mathbf{r}_{i}^{\dagger}\right)$ by averaging on received samples.

Step 2. Find $q=2(J M-K N)$ coorthogonal eigenvectors, $\boldsymbol{\beta}_{k}(k=0,1, \ldots, q-1)$, corresponding to the smallest $q$ eigenvalues of matrix $\mathbf{R}_{r}$.

Step 3.Form the matrix $\mathbf{G}$ defined in (37) from $\boldsymbol{\alpha}_{k}=\mathbf{P} \boldsymbol{\beta}_{k}$, where $\mathbf{P}$ is the constant permutation matrix defined in (16), and compute the SVD of $\mathbf{G}$. Choose the $2 K$ right singular vectors corresponding to the $2 K$ smallest singular values. Use the $2 K$ singular vectors as columns to create a matrix $\mathbf{V}$ of size $2 J(L+1) \times 2 K$. Divide $\mathbf{V}$ into blocks as

$$
\mathbf{V}=\left[\begin{array}{llll}
\mathbf{V}^{T}(0) & \mathbf{V}^{T}(1) & \cdots & \mathbf{V}^{T}(2 L+1)
\end{array}\right]^{T}
$$

where $\mathbf{V}(l)$ are $J \times 2 K$ matrices. Then the channels are

$\mathbf{h}_{1}(l)=\mathbf{V}(2 l) \mathbf{C}$

$\mathbf{h}_{2}(l)=\mathbf{V}(2 l+1) \mathbf{C}, \quad l=0,1, \ldots, L$

where $\mathbf{C}$ is a $2 K \times K$ constant matrix which cannot be determined by the subspace method.

\section{EQUALIZATION}

Once the channels have been estimated, the MIMO STCOFDM system can be equalized by using the fast Fourier transform (FFT). Compared to the conventional OFDM, the STC- 
OFDM is less sensitive to channel nulls [7], [11]. It is well known that a major problem of OFDM is the channel nulls problem. If the channel nulls hit a subcarrier, the frequency domain per-tone equalization will be very unreliable at this subcarrier. For the STC-OFDM [7], even if the nulls of one channel hit a subcarrier but the nulls of another channel do not hit this same subcarrier, the per-tone equalization is still reliable for this subcarrier.

Unlike the equalization of CP-OFDM, where the first $L$ elements of each received block is discarded, per-tone equalization of ZP-OFDM requires the received signal to be first overlap added before the DFT [25]. Received blocks are overlap added as

$$
\hat{\mathbf{x}}_{i}(n)= \begin{cases}\mathbf{x}_{i}(n)+\mathbf{x}_{i}(n+N) & n=0,1, \ldots, L-1 \\ \mathbf{x}_{i}(n), & n=L, L+1, \ldots, N-1 .\end{cases}
$$

Each overlap added block is then transformed by the DFT. Let $\mathbf{y}_{i}(n)$ be the DFT of $\hat{\mathbf{x}}_{i}(n)(n=0,1, \ldots, N-1)$. Zero-pad channel $\mathbf{h}_{j}(l)$ to length $N$ and let the DFT (length $N$ ) of the channels be $\mathrm{g}_{j}(n)$. Then from (1) and (2) it is easy to prove that

$$
\begin{aligned}
\mathbf{y}_{2 i}(n) & =\mathbf{g}_{1}(n) \mathbf{s}_{2 i}(n)+\mathbf{g}_{2}(n) \mathbf{s}_{2 i+1}(n)+\zeta_{2 i}(n) \\
\mathbf{y}_{2 i+1}^{*}(n) & =\mathbf{g}_{2}^{*}(n) \mathbf{s}_{2 i}(n)-\mathbf{g}_{1}^{*}(n) \mathbf{s}_{2 i+1}(n)+\zeta_{2 i+1}^{*}(n) \\
n & =0,1, \ldots, N-1
\end{aligned}
$$

where $\boldsymbol{\zeta}_{i}(n)$ is the noise after the DFT and $\mathbf{s}_{i}(n)=$ $\left[s_{i}^{(1)}(n) \quad \cdots \quad s_{i}^{(K)}(n)\right]^{T}$. Defining a matrix

$$
\mathbf{T}(n)=\left[\begin{array}{cc}
\mathbf{g}_{1}(n) & \mathbf{g}_{2}(n) \\
\mathbf{g}_{2}^{*}(n) & -\mathbf{g}_{1}^{*}(n)
\end{array}\right]
$$

we obtain a least square (LS) estimation for the symbols as

$$
\left[\begin{array}{c}
\mathbf{s}_{2 i}(n) \\
\mathbf{s}_{2 i+1}(n)
\end{array}\right]=\left(\mathbf{T}^{\dagger}(n) \mathbf{T}(n)\right)^{-1} \mathbf{T}^{\dagger}(n)\left[\begin{array}{c}
\mathbf{y}_{2 i}(n) \\
\mathbf{y}_{2 i+1}^{*}(n)
\end{array}\right] .
$$

Especially when $J=K=1$ (one user, two transmitting antennas, and one receiving antenna), $\mathbf{s}_{i}(n), \mathbf{y}_{i}(n)$, and $\mathbf{g}_{1}(n)$, $\mathrm{g}_{2}(n)$ are scalars and, therefore

$$
\begin{aligned}
\left(\mathbf{T}^{\dagger}(n) \mathbf{T}(n)\right)^{-1} \mathbf{T}^{\dagger}(n)=\frac{1}{\left|\mathbf{g}_{1}(n)\right|^{2}+\left|\mathbf{g}_{2}(n)\right|^{2}} \\
\times\left[\begin{array}{cc}
\mathbf{g}_{1}^{*}(n) & \mathbf{g}_{2}(n) \\
\mathbf{g}_{2}^{*}(n) & -\mathbf{g}_{1}(n)
\end{array}\right]
\end{aligned}
$$

Equation (44) means that the symbols can be recovered if at least one DFT coefficient of the two channels $\left(\mathrm{g}_{1}(n)\right.$ or $\left.\mathrm{g}_{2}(n)\right)$ is not zero, that is, the STC-OFDM achieves a diversity gain of order two [7], [11]. A linear minimum mean square error (mmse) estimation can also be constructed as follows.

$$
\begin{aligned}
{\left[\begin{array}{c}
\mathbf{s}_{2 i}(n) \\
\mathbf{s}_{2 i+1}(n)
\end{array}\right]=\mathbf{T}^{\dagger}(n)\left(\mathbf{T}(n) \mathbf{T}^{\dagger}(n)+\right.} & \left.\frac{\sigma_{\eta}^{2}}{\sigma_{s}^{2}} \mathbf{I}_{2 M}\right)^{-1} \\
& \times\left[\begin{array}{c}
\mathbf{y}_{2 i}(n) \\
\mathbf{y}_{2 i+1}^{*}(n)
\end{array}\right]
\end{aligned}
$$

where $\sigma_{s}^{2}$ is the variance of transmitted symbols.

\section{RESOLVING THE AMBIGUITY}

Now we consider resolving the ambiguity matrix $\mathbf{C}$ in (39). If the noise terms are ignored, (41) can be rewritten as

$$
\begin{aligned}
{\left[\begin{array}{ll}
\mathbf{y}_{2 i}(n) & \mathbf{y}_{2 i+1}(n)
\end{array}\right]=\left[\begin{array}{ll}
\mathbf{g}_{1}(n) & \mathbf{g}_{2}(n)
\end{array}\right] } \\
\times\left[\begin{array}{cc}
\mathbf{s}_{2 i}(n) & -\mathbf{s}_{2 i+1}(n) \\
\mathbf{s}_{2 i+1}(n) & \mathbf{s}_{2 i}(n)
\end{array}\right] .
\end{aligned}
$$

If we fix the index $n=0$ and group $N_{p}$ equations together, we obtain

$$
\mathbf{Y}=\left[\begin{array}{ll}
\mathbf{g}_{1}(0) & \mathbf{g}_{2}(0)
\end{array}\right] \mathbf{S}
$$

where $\mathbf{S}$ and $\mathbf{Y}$ are $2 K \times 2 N_{p}$ and $J \times 2 N_{p}$ matrices, respectively, defined as

$$
\begin{aligned}
\mathbf{S} & =\left[\begin{array}{ccccc}
\mathbf{s}_{0}(0) & -\mathbf{s}_{1}(0) & \cdots & \mathbf{s}_{2 N_{p}-2}(0) & -\mathbf{s}_{2 N_{p}-1}(0) \\
\mathbf{s}_{1}(0) & \mathbf{s}_{0}(0) & \cdots & \mathbf{s}_{2 N_{p}-1}(0) & \mathbf{s}_{2 N_{p}-2}(0)
\end{array}\right] \\
\mathbf{Y} & =\left[\begin{array}{lllll}
\mathbf{y}_{0}(0) & \mathbf{y}_{1}(0) & \cdots & \mathbf{y}_{2 N_{p}-2}(0) & \mathbf{y}_{2 N_{p}-1}(0)
\end{array}\right] .
\end{aligned}
$$

If $\mathbf{s}_{i}(0)\left(i=0,1, \ldots, 2 N_{p}-1\right)$ are pilot symbols and $N_{p} \geq K$, we obtain an LS solution for $\mathbf{g}_{1}(0)$ and $\mathbf{g}_{2}(0)$ as

$$
\left[\begin{array}{ll}
\mathbf{g}_{1}(0) & \mathbf{g}_{2}(0)
\end{array}\right]=\mathbf{Y S}^{\dagger}\left(\mathbf{S S}^{\dagger}\right)^{-1} \text {. }
$$

Since $\mathbf{g}_{1}(n)$ and $\mathbf{g}_{2}(n)$ are the DFTs of $\mathbf{h}_{1}(l)$ and $\mathbf{h}_{2}(l)$ (zeropadded to length $N)$, respectively, it is clear that

$$
\mathrm{g}_{1}(0)=\sum_{l=0}^{L} \mathbf{h}_{1}(l), \quad \mathbf{g}_{2}(0)=\sum_{l=0}^{L} \mathbf{h}_{2}(l) .
$$

Noticing that $\mathbf{h}_{1}(l)=\mathbf{V}(2 l) \mathbf{C}$ and $\mathbf{h}_{2}(l)=\mathbf{V}(2 l+1) \mathbf{C}$, we obtain

$$
\left[\begin{array}{l}
\mathbf{g}_{1}(0) \\
\mathbf{g}_{2}(0)
\end{array}\right]=\mathbf{W C}
$$

where

$$
\mathbf{W}=\left[\begin{array}{c}
\sum_{l=0}^{L} \mathbf{V}(2 l) \\
\sum_{l=0}^{L} \mathbf{V}(2 l+1)
\end{array}\right]
$$

Finally, the matrix $\mathbf{C}$ is found as

$$
\mathbf{C}=\left(\mathbf{W}^{\dagger} \mathbf{W}\right)^{-1} \mathbf{W}^{\dagger}\left[\begin{array}{l}
\mathbf{g}_{1}(0) \\
\mathbf{g}_{2}(0)
\end{array}\right]
$$

In whole, if each user sends $2 N_{p}\left(N_{p} \geq K\right)$ pilot symbols, the ambiguity matrix can be determined and therefore the channels are estimated. On the contrary, if purely pilot-based method is used, each user must send $2 K(L+1)$ pilot symbols for estimating the channels [31].

\section{Simulations AND Discussions}

In practice, the autocorrelation matrix $\mathbf{R}_{r}$ can only be approximated from the output samples. Although there may be better methods to estimate it, here we use a standard method as $\mathbf{R}_{r}=\left(1 / N_{s}\right) \sum_{i=0}^{N_{s}-1} \mathbf{r}_{i} \mathbf{r}_{i}^{\dagger}$, where $N_{s}$ is the number of block samples used. The noise variance is estimated by averaging the $q$ least eigenvalues of $\mathbf{R}_{r}$. As usual, the signal-to-noise ratio 


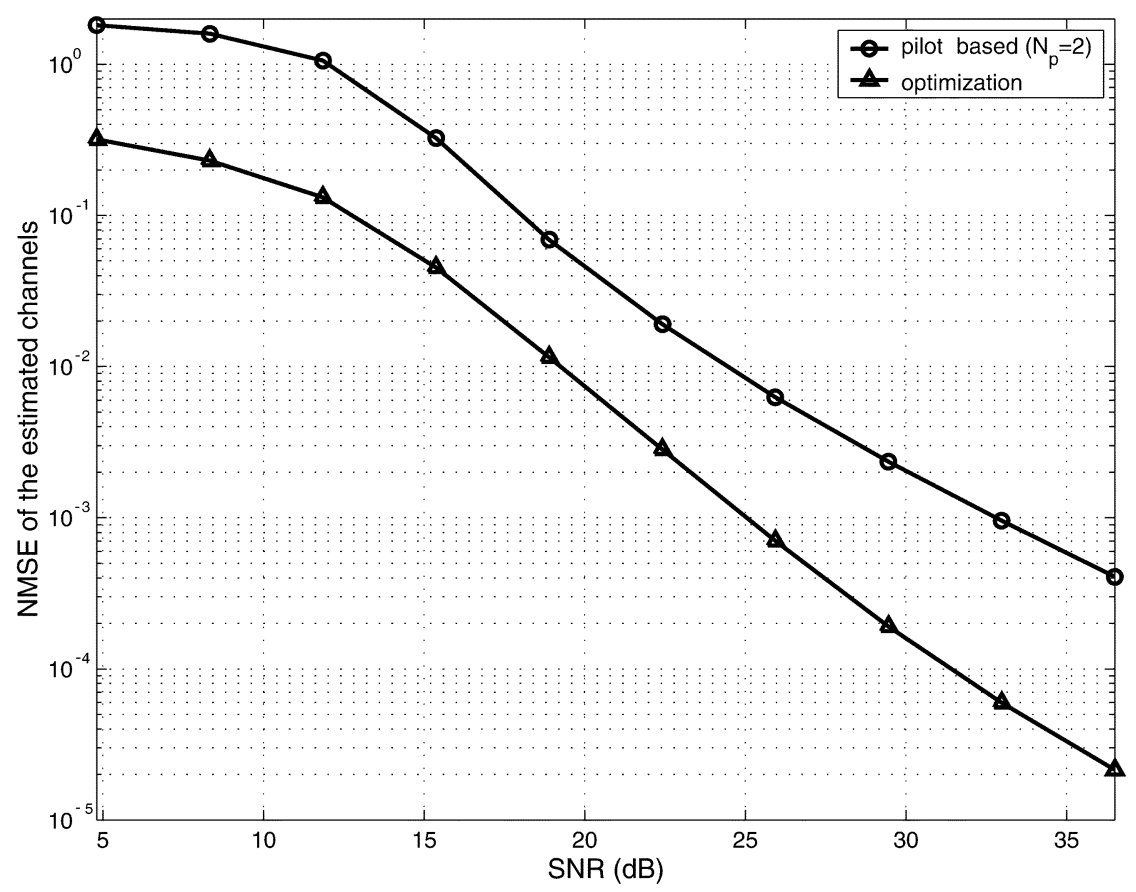

Fig. 2. NMSE versus SNR $(K=2, J=3)$.

(SNR) means the ratio of the average received signal power to the average noise power

$$
\mathrm{SNR}=\frac{\mathrm{E}\left(\sum_{n=0}^{M-1}\left\|\mathbf{x}_{i}(n)-\boldsymbol{\eta}_{i}(n)\right\|^{2}\right)}{\mathrm{E}\left(\sum_{n=0}^{M-1}\left\|\boldsymbol{\eta}_{i}(n)\right\|^{2}\right)} .
$$

The normalized mean square error (NMSE) (averaging on all channels) between the estimated and true channel responses is defined as

$$
\mathrm{NMSE}=\frac{\sum_{j=1}^{2} \sum_{l=0}^{L}\left\|\tilde{\mathbf{h}}_{j}(l)-\mathbf{h}_{j}(l)\right\|_{F}^{2}}{\sum_{j=1}^{2} \sum_{l=0}^{L}\left\|\mathbf{h}_{j}(l)\right\|_{F}^{2}}
$$

where $\tilde{\mathbf{h}}_{j}(l)$ and $\mathbf{h}_{j}(l)$ are the estimated (without ambiguity) and true channel responses, respectively, and $\|\cdot\|_{F}$ is the Frobenius norm for matrix.

\section{A. Simulations}

Since the determination of the ambiguities is unrelated to the subspace method, it is logical to use the best estimation of the ambiguities to compute the NMSE for evaluating the subspace method. The best ambiguity matrix $\mathbf{C}$ can be obtained from optimization using the true channels, which can be expressed explicitly as

$$
\begin{aligned}
\mathbf{C}=\left(\sum_{l=0}^{2 L+1} \mathbf{V}^{\dagger}(l) \mathbf{V}(l)\right)^{-1} \\
\quad \times\left(\sum_{l=0}^{L}\left(\mathbf{V}^{\dagger}(2 l) \mathbf{h}_{1}(l)+\mathbf{V}^{\dagger}(2 l+1) \mathbf{h}_{2}(l)\right)\right) .
\end{aligned}
$$

Using such a means to get the ambiguity matrix is of course not practical, however, it best describes the performance of the subspace method and tells us the gap between the pilot-based and best ambiguity matrix. So, in the following, two NMSE/BER curves are usually given, one corresponds to the ambiguities resolved by the pilot-based method and the other by optimization. Extensive simulations have been done to verify the effectiveness of the method. Two examples are given below. No channel coding is considered for all the examples.

Example 1: A multiuser STC-OFDM system ( $K=2$ and $J=3$ ) is considered here. The transmitted baseband signals are BPSK. Other parameters are: $N=32, L=7$, and $N_{p}=2$ (the number of pilot symbols is $2 N_{p}$ ). For each Monte Carlo realization, 12 random Rayleigh fading channels (for simplicity, we assume that the tap coefficients are statistically independent and have the same complex Gaussian distribution) with channel orders not greater than seven are created, and inputs and noises are generated randomly.

Fig. 2 shows the NMSEs versus SNRs, where $N_{s}=200$. When the SNR is fixed to $25 \mathrm{~dB}$, the NMSEs are shown in Fig. 3 with $N_{s}$ varying from 140 to 240 . From the figures, we see that the semiblind method works well. However, they also show that the pilot-based method usually cannot get the best values for the ambiguities, which means that there is room to improve the pilot-based method for resolving the ambiguities. The main reason is that the matrix $\mathbf{W}$ defined in (53) may be ill-conditioned. It is also clear that larger sample size gives smaller NMSE.

The BERs versus SNRs are shown in Fig. 4 when $N_{s}=200$. The mmse method is used for equalization. Here the results are averaged over 500 Monte Carlo realizations. For each Monte Carlo realization, 400 block symbols of each user are equalized. For comparison, the BERs using true channel responses are also shown (the line without marks). Since the test is based on finite 


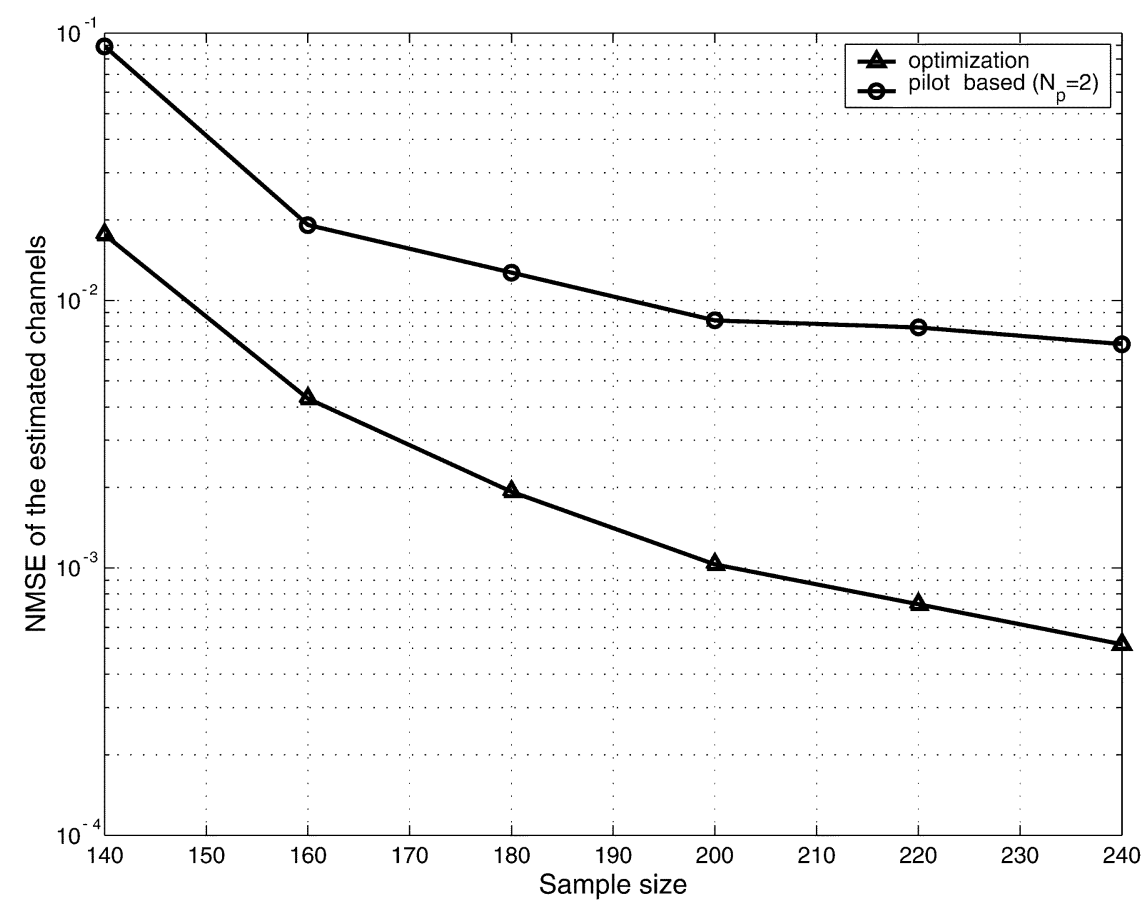

Fig. 3. NMSE versus sample size $(K=2, J=3)$.

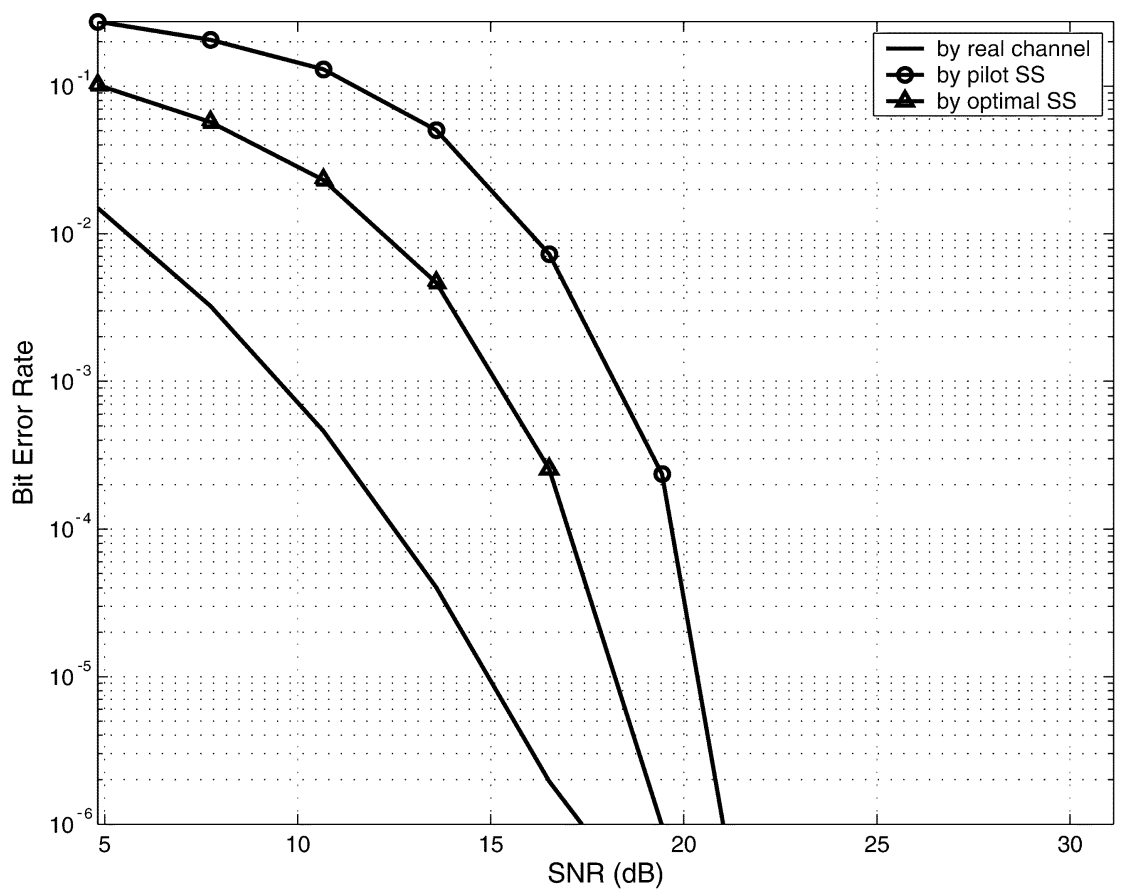

Fig. 4. BER versus SNR $(K=2, J=3)$.

number of samples, very small $\left(<10^{-6}\right)$ BERs are meaningless and therefore they are not shown. From the figure, it is clear the accuracy of channel estimation is vital to the BER performance.

Figs. 5 and 6 show the NMSEs and BERs, respectively, when the transmitted power for user 1 is $10 \mathrm{~dB}$ lower than that for user 2 (or the channel fading for user 1 is $10 \mathrm{~dB}$ worse than that for user 2). Since the signal for user 1 is much weaker, the NMSEs and BERs for user 1 are much worse than those for user 2. Even if the real (true) channels are used for equalization, the BERs for user 1 are still much worse, that is, the linear LS or mmse equal- ization itself is also vulnerable to the "near-far effect" (some nonlinear methods may be used for better performance at the expense of higher complexity).

Example 2: We consider a single-user $(K=1$ and $J=1)$ STC-OFDM system with "bad" channels (not coprime channels). The transmitted baseband signals are BPSK and the OFDM block length $N=32$. The two channels are generated deliberately such that they are not coprime. The two transfer functions are $h_{1}(z)=\left(z^{-1}-\sqrt{2}-\sqrt{2} i\right)[0.8644+$ $0.3962 i+(0.0942-0.9649 i) z^{-1}+(-0.8519+0.1684 i) z^{-2}+$ 


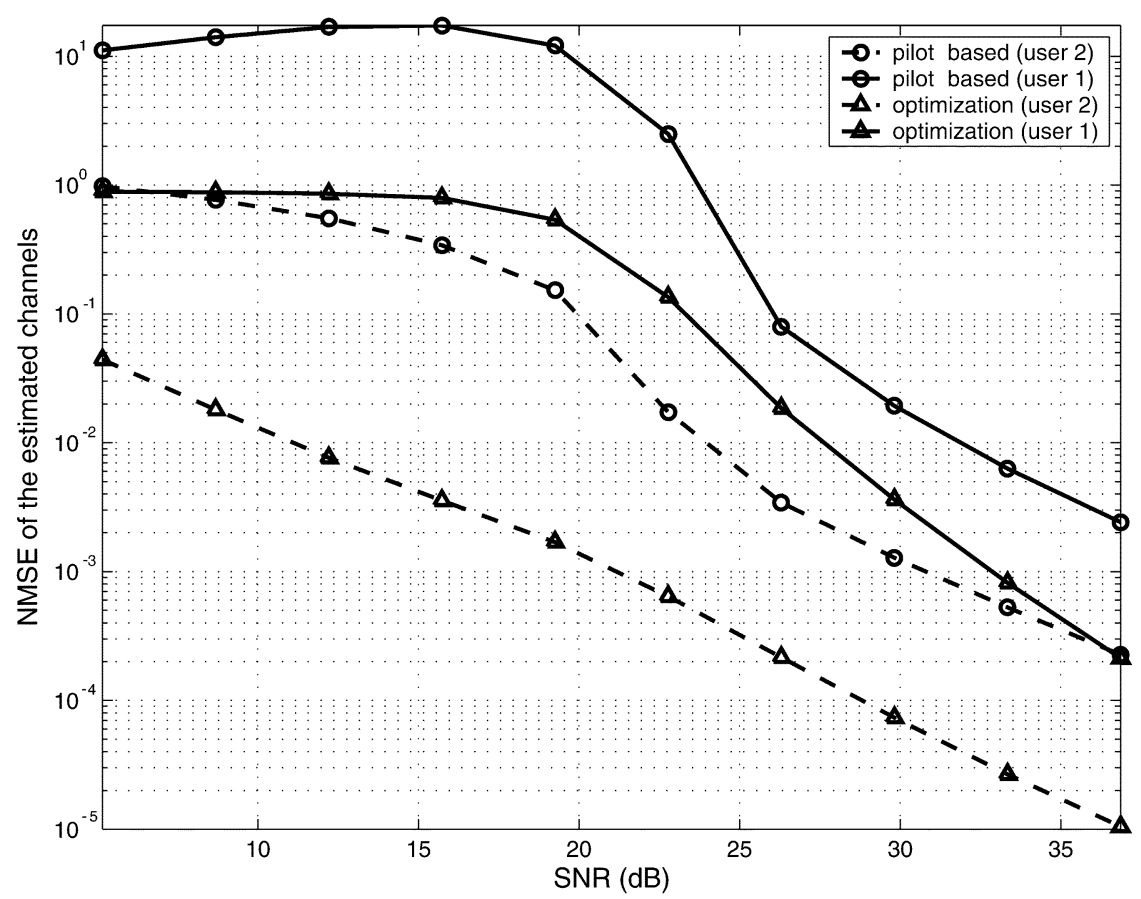

Fig. 5. NMSE versus SNR $(K=2, J=3)$.

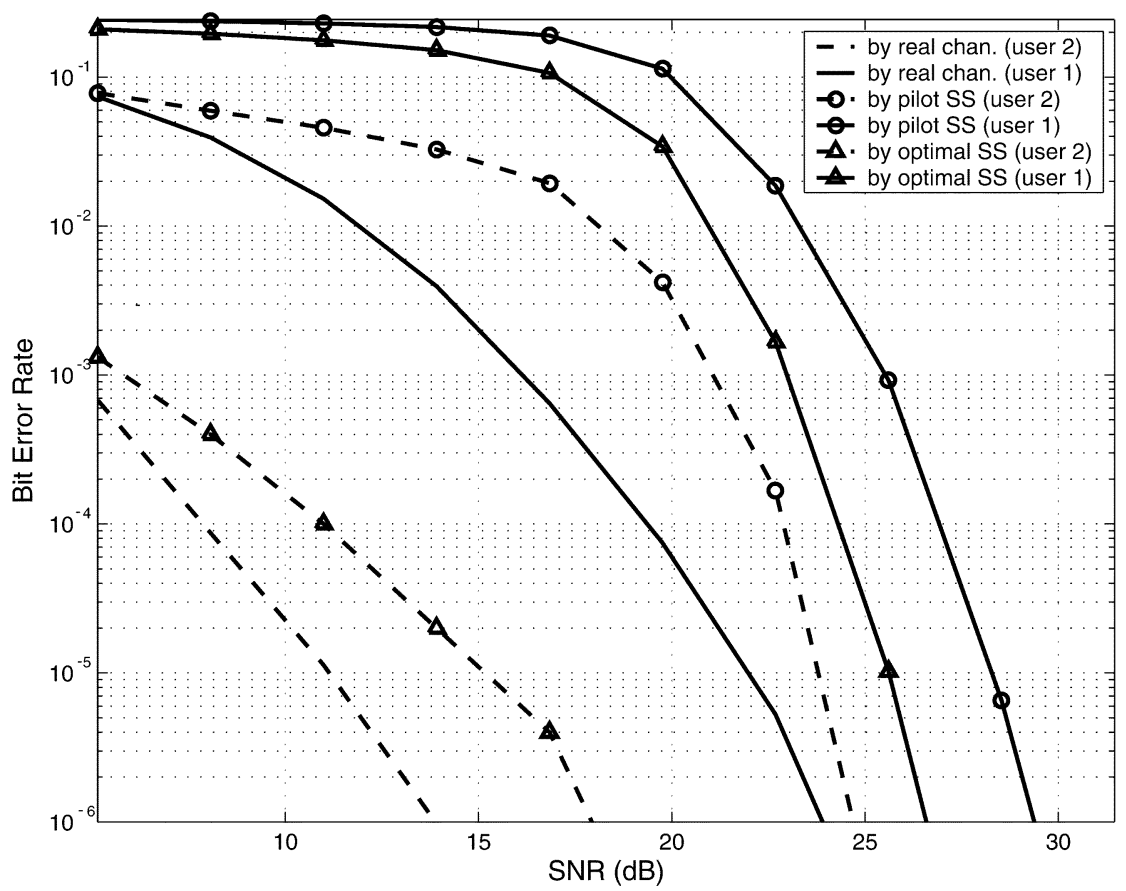

Fig. 6. BER versus SNR $(K=2, J=3)$.

$(0.8735-1.9654 i) z^{-3}+(-0.4380-0.7443 i) z^{-4}+(-0.4297-$ $\left.0.5523 i) z^{-5}+(-1.1027-0.8197 i) z^{-6}\right]$ and $h_{2}(z)=$ $\left(z^{-1}-\sqrt{2}-\sqrt{2} i\right)[1.1091-0.8661 i+(-0.6149-$ $2.1165 i) z^{-1}+(-0.2546-0.9645 i) z^{-2}+(-0.2698+$ $0.2127 i) z^{-3}+(-1.6720+0.4779 i) z^{-4}+(-1.8760+$ $\left.0.1007 i) z^{-5}+(0.5750+0.2974 i) z^{-6}\right]$, respectively.

Fig. 7 shows the NMSEs versus the SNRs $\left(N_{s}=90\right.$ and $N_{p}=1$ ). The BERs versus SNRs are shown in Fig. 8. The results here are averaged over 1000 Monte Carlo realizations on inputs and noises, while for each realization 200 OFDM blocks are generated randomly. It is seen that the semiblind and optimal subspace methods still work well even if the channels are not coprime.

\section{B. Discussions}

The proposed method inherits some advantages of the subspace technique, such as simple structure and good performance. Sensitive to order overestimation is a major drawback of some known subspace methods in wireless communication [17], [18], [27], [28], [30], but here this drawback has been 


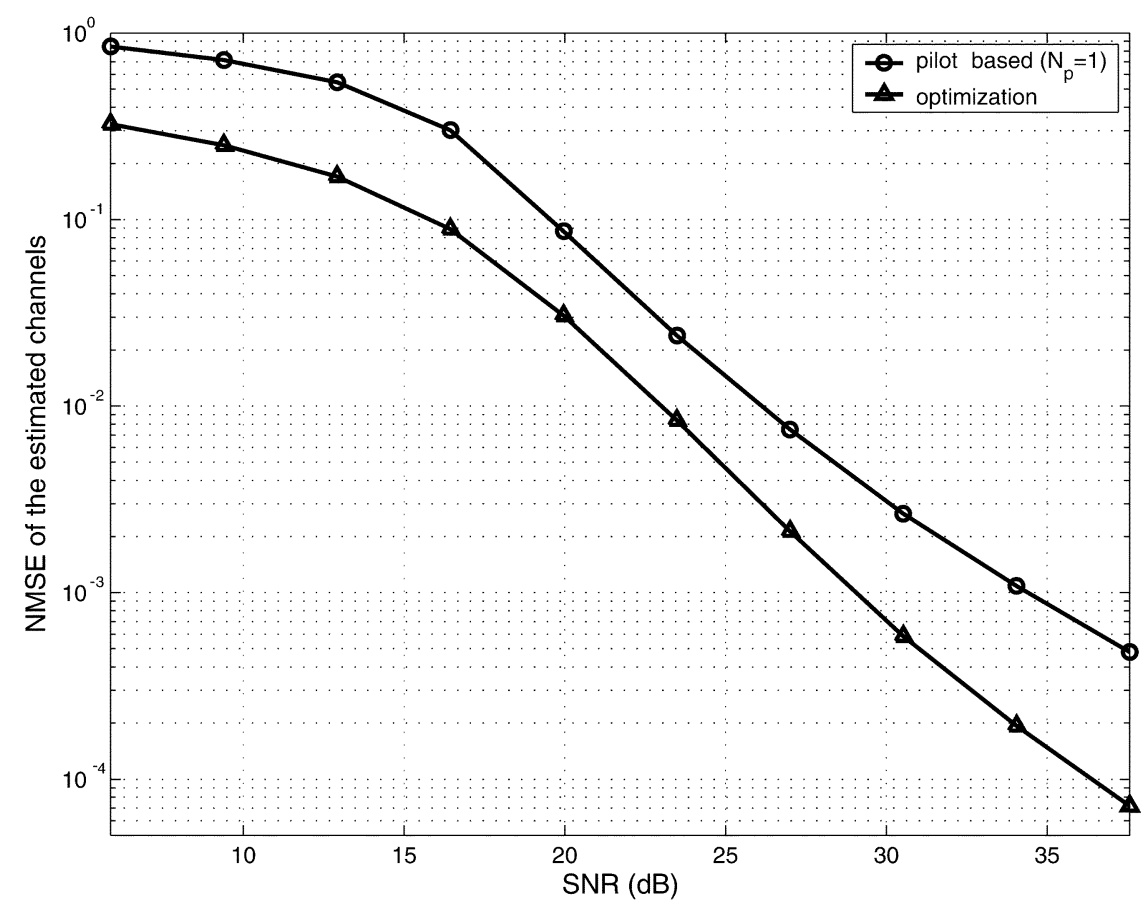

Fig. 7. NMSE versus SNR ( $K=1, J=1$, not coprime channels).

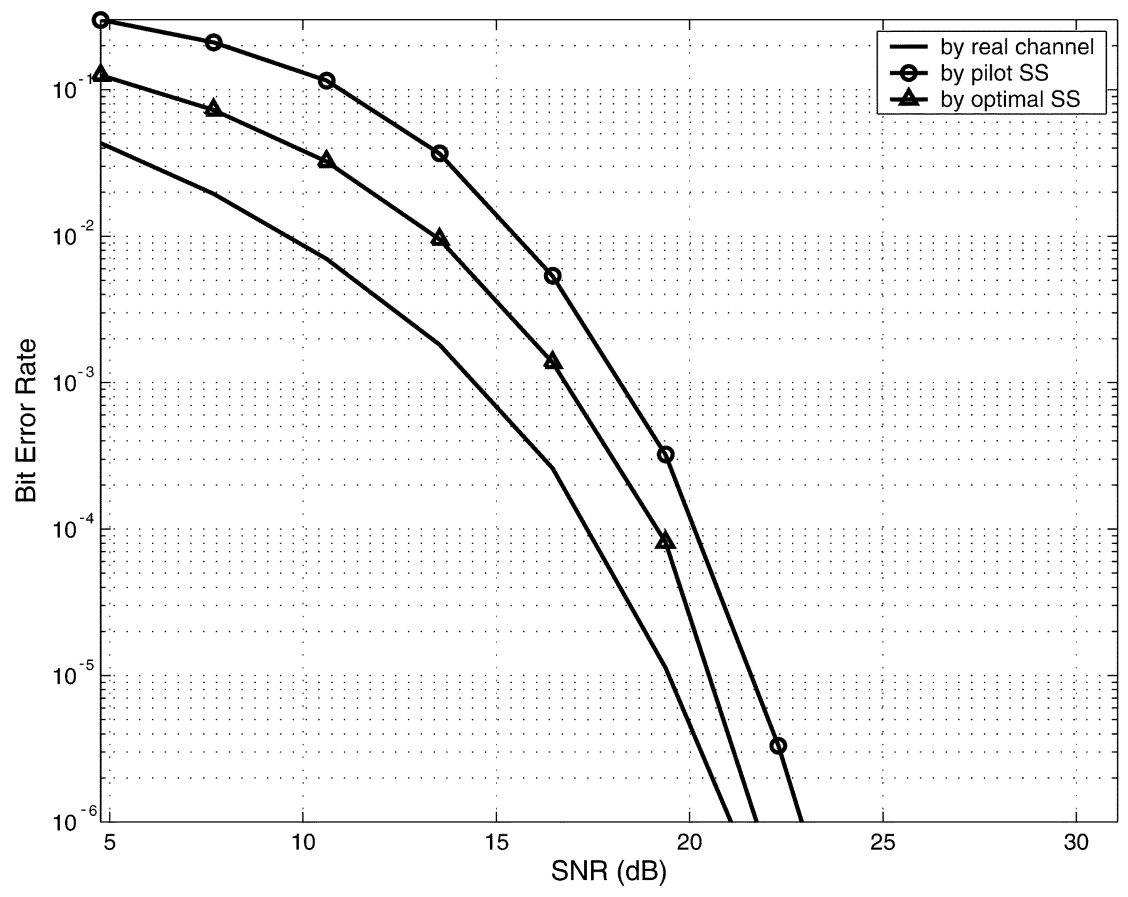

Fig. 8. BER versus SNR ( $K=1, J=1$, not coprime channels).

overcome (only an upper bound for all the channel orders is required for the proposed method) due to the special structure of STC and ZP-OFDM. Compared to known blind (semiblind) channel estimation/equalization methods for STC-OFDM [7], [11], [21], [22], the proposed method has some merits. First, methods in [7] and [11] are only valid for single user case with two transmitting antennas and one receiving antenna, while the proposed method is applicable for multiuser MIMO STC-OFDM systems with multiple transmitting and receiving antennas. Channel estimation for multiuser MIMO
STC-OFDM is usually much more difficult because the number of channels increases rapidly with the number of antennas and users. Second, compared to the method in [7], the proposed one eliminates two constraints: coprime transfer functions and constant modulus transmit signals. Compared to the method in [11], no precoding is needed in the proposed method and therefore bandwidth is saved and system complexity is reduced. Third, the identifiability of the methods in [21]-[23] is not guaranteed while it is mathematically proved in the proposed method. However, the method does have a drawback: it requires 
the input symbols to be real or symmetric. When the input is complex without symmetry, (1) and (2) are changed. Yet we have not found any theoretical proof for the identifiability of the subspace-based method at this case.

If the system uses the CP-OFDM other than the ZP-OFDM, it is more difficult to estimate the channels since there is inter block interferences. A subspace method for single-user singleantenna CP-OFDM system is proposed in [29]. It is possible (but seems not easy) to extend the method to MIMO STC-OFDM systems.

\section{CONCLUSION}

A subspace-based blind method has been proposed for estimating the channel responses of an MIMO STC-OFDM system. It is mathematically proved that all channel responses can be identified subject to two ambiguity matrices. Unlike some subspace methods in wireless communications, the proposed method does not need precise channel order information (only requires an upper bound for the orders) and does not subject to the constraint of channel coprime. Furthermore, a method has been proposed to resolve the ambiguities by using few pilot symbols.

\section{ACKNOWLEDGMENT}

The authors thank the editors and the anonymous reviewers for their invaluable comments.

\section{REFERENCES}

[1] N. Al-Dhahir, C. Fragouli, A. Stamoulis, W. Younis, and R. Calderbank, "Space-time processing for broadband wireless access," IEEE Commun. Mag., no. 9, pp. 136-142, Sep. 2002.

[2] D. Gesbert, M. Shafi, D. S. Shiu, P. J. Smith, and A. Naguib, "From theory to practice: An overview of MIMO space-time coded wireless systems," IEEE J. Select. Areas Commun., vol. 21, pp. 281-302, 2003.

[3] S. M. Alamouti, "A simple transmit diversity technique for wireless communications," IEEE J. Sel. Areas Commun., vol. 16, pp. 1451-1458, 1998.

[4] N. Al-Dhahir, "Single-carrier frequency-domain equalization for spacetime block-coded transmissions over frequency-selective fading channels," IEEE Commun. Lett., no. 7, pp. 304-306, Jul. 2001.

[5] E. Lindskog and A. Paulraj, "A transmit diversity scheme for channel with intersymbol interference," in Proc. Int. Conf. Commun. (ICC), vol. 1, 2000, pp. 307-311

[6] Z. Liu and G. B. Giannakis, "Space-time block coded multiple access through frequency-selective fading channels," IEEE Trans. Commun., vol. 49, pp. 1033-1044, 2001.

[7] Z. Liu, G. B. Giannakis, S. Barbarossa, and A. Scaglione, "Transmit antennae space-time block coding for generalized OFDM in the presence of unknown multipath," IEEE J. Sel. Areas Commun., vol. 19, pp. 1352-1364, 2001.

[8] V. Tarokh, N. Seshadri, and A. R. Calderbank, "Space-time codes for high data rate wireless communications: Performance criterion and code construction," IEEE Trans. Inf. Theory, vol. 44, no. 2, pp. 744-765, Mar. 1998

[9] V. Tarokh, H. Jafarkhani, and A. R. Calderbank, "Space-time block codes from orthogonal designs," IEEE Trans. Inf. Theory, vol. 45, no. 4, pp. 1456-1467, Jul. 1999.

[10] J. Chuang and N. Sollenberger, "Beyond 3G: Wideband wireless data access based on OFDM and dynamic packet assignment," IEEE Commun. Mag., vol. 32, no. 1, pp. 78-87, 2000.

[11] S. Zhou, B. Muquet, and G. B. Giannakis, "Subspace-based (semi-) blind channel estimation for block precoded space-time OFDM," IEEE Trans. Signal Process., vol. 50, pp. 1215-1228, 2002.

[12] B. M. Hochwald and T. L. Marzetta, "Unitary space-time modulation for multiple-antenna communications in Rayleigh flat fading," IEEE Trans. Inf. Theory, vol. 46, pp. 543-564, 2000.
[13] B. M. Hochwald and W. Sweldens, "Differential unitary space-time modulation," IEEE Trans. Commun., vol. 48, pp. 2041-2052, Dec. 2000.

[14] B. L. Hughes, "Differential space-time modulation," IEEE Trans. Inf. Theory, vol. 46, pp. 2567-2578, Nov. 2000

[15] V. Tarokh and H. Jafarkhani, "A differential detection scheme for transmit diversity," IEEE J. Sel. Areas Commun., vol. 18, pp. 1169-1174, Jul. 2000.

[16] C. Budianu and L. Tong, "Channel estimation for space-time orthogonal block codes," IEEE Trans. Signal Process., vol. 50, pp. 2515-2528, 2002.

[17] Z. Ding and Y. Li, Blind Equalization and Identification. New York: Marcel Dekker, 2001.

[18] G. B. Giannakis, Y. Hua, P. Stoica, and L. Tong, Signal Processing Advances in Wireless \& Mobile Communications. Englewood Cliffs, NJ Prentice-Hall PTR, 2001, vol. 1.

[19] E. G. Larsson, P. Stoica, and J. Li, "Orthogonal space-time block codes: Maximum likelihood detection for unknown channels and unstructured interferences," IEEE Trans. Signal Process., vol. 51, pp. 362-372, 2003.

[20] S. Shahbazpanahi, A. B. Gershman, and J. H. Manton, "Closed-form blind decoding of orthogonal space-time block codes," in Proc. IEEE ICASSP Conf., Canada, 2004.

[21] A. L. Swindlehurst and G. Leus, "Blind and semi-blind equalization for generalized space-time block codes," IEEE Trans. Signal Process., vol. 50, pp. 2489-2498, 2002.

[22] G. Leus and M. Moonen, "Per-tone equalization for MIMO OFDM systems," IEEE Trans. Signal Process., vol. 51, pp. 2965-2975, 2003.

[23] J. Choi, "Equalization and semi-blind channel estimation for space-time block coded signals over a frequency-selective fading channel," IEEE Trans. Signal Process., vol. 52, pp. 774-785, 2004.

[24] Y. H. Zeng and T. S. Ng, "A semi-blind channel estimation method for multi-user multi-antenna OFDM systems," IEEE Trans. Signal Process. vol. 52, pp. 1419-1429, 2004.

[25] A. Scaglione, G. B. Giannakis, and S. Barbarossa, "Redundant filterbank precoders and equalizers-part II: Blind channel estimation, synchronization and direct equalization," IEEE Trans. Signal Process., vol. 47, pp. 2007-2022, 1999 .

[26] B. Muquet, Z. Wang, G. B. Giannakis, M. D. Courville, and P. Duhamel, "Cyclic prefixing or zero padding for wireless multicarrier transmissions," IEEE Trans. Commun., vol. 50, no. 12, pp. 2136-2148, 2002.

[27] E. Moulines, P. Duhamel, J. F. Cardoso, and S. Mayrargue, "Subspace methods for the blind identification of multichannel FIR filters," IEEE Trans. Signal Process., vol. 43, pp. 516-525, 1995.

[28] K. Abed-Meraim, P. Loubaton, and E. Moulines, "A subspace algorithm for certain blind identification problem," IEEE Trans. Inf. Theory, vol. 32, no. 2, pp. 499-511, 1997.

[29] B. Muquet, M. D. Courville, and P. Duhamel, "Subspace-based blind and semi-blind channel estimation for OFDM systems," IEEE Trans. Signal Process., vol. 50, pp. 1699-1712, 2002.

[30] S. Roy and C. Y. Li, "A subspace blind channel estimation method for OFDM systems without cyclic prefix," IEEE Trans. Wireless Commun., vol. 1, no. 4, pp. 572-579, 2002.

[31] Y. Li, J. H. Winters, and N. R. Sollenberger, "MIMO-OFDM for wireless communications: Signal detection with enhanced channel estimation," IEEE Trans. Commun., vol. 50, pp. 1471-1477, 2002.

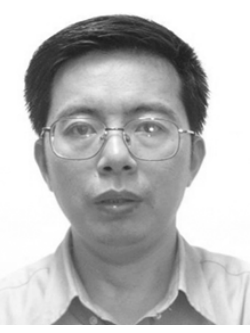

Yonghong Zeng (M'99-SM'05) received the B.S degree in mathematics from the Peking University, Beijing, China, and the M.S. degree in applied mathematics and the Ph.D. degree in computer science from the National University of Defense Technology, Changsha, China.

$\mathrm{He}$ worked as an Associate Professor with the National University of Defense Technology until July 1999. From August 1999 to October 2004, he was a Research Fellow with the Nanyang Technological University, Singapore, and the University of Hong Kong, successively. Since November 2004, he has been with the Institute for Infocomm Research, A-STAR, Singapore, working as a scientist. His current research interests include signal processing and wireless communication, especially channel estimation, equalization, detection, synchronization, cognitive radio, and software defined radio. He has coauthored six books, including Transforms and Fast Algorithms for Signal Analysis and Representation (Boston, MA: Birkhäuser, 2003) and more than 50 refereed journal papers.

Dr. Zeng received the ministry-level Scientific and Technological Development Awards in China four times. 


$$
88
$$

